\title{
Influência de fatores exógenos e endógenos e da evidenciação dos Objetivos de Desenvolvimento Sustentável no desempenho de companhias abertas*
}

\section{Francieli Pacassa, Sady Mazzioni, Cristian Baú Dal Magro, André Casagrande Medeiros}

Universidade Comunitária da Região de Chapecó (Unochapecó) - Brasil

Apesar do desenvolvimento de estudos sobre evidenciação social e ambiental pela comunidade científica, pouco se tem avançado sobre a incorporação dos Objetivos de Desenvolvimento Sustentável (ODS) como elementos de evidenciação, se fatores endógenos (tamanho e nível de governança corporativa) e exógenos (investimentos em carteiras sustentáveis e internacionalizaçáo) afetam de forma diferente o nível de evidenciação dos ODS, se há uma influência direta e indireta destes fatores sobre o desempenho. Nessa perspectiva, esta pesquisa analisa se fatores exógenos e endógenos e a evidenciação relacionada aos ODS afetam o desempenho em companhias abertas listadas na bolsa de valores Brasil, Bolsa, Balcáo - $[\mathrm{B}]^{3}$. Para tanto, realizou-se pesquisa quantitativa, de caráter descritivo e por meio de coleta de dados documental em relatórios de sustentabilidade e banco de dados da Economatica ${ }^{\circledR}$, no período de 2016 a 2018. A partir dos relatórios, foi realizada uma análise de conteúdo, obtendo o número de palavras associadas aos ODS, das quais se originou o índice do nível de evidenciação. Ademais, a análise e validação dos dados se deram pela utilização da regressão linear múltipla e demais técnicas estatísticas pertinentes. Os resultados indicam que fatores endógenos, exógenos e a evidenciação dos ODS influenciam o Market-to-Book das empresas.

Palavras-chave: evidenciação, ODS, desempenho de mercado, companhias abertas

\section{Influence of exogenous and endogenous factors and disclosure of the SDG on the performance of public companies}

Despite the development of studies on social and environmental disclosure by the scientific community, little progress has been made on the incorporation of sustainable development goals (SDGs) as elements of disclosure, whether endogenous (size and level of corporate governance) and exogenous factors (investments in sustainable portfolios and internationalization) affect the level of disclosure of the SDGs differently, if there is a direct and indirect influence of these factors on performance. In this perspective, this research aims to analyze whether exogenous and endogenous factors and disclosure related to the SDGs affect performance in public companies listed in Brasil, Bolsa, Balcão - $[\mathrm{B}]^{3}$. To this end, quantitative research was carried out, of a descriptive nature and by means of documentary data collection in sustainability reports / integrated reports and Economatica database, in the period from 2016 to 2018. Based on the reports, a content analysis was performed, obtaining the number of words associated with the SDGs, from which the disclosure level index originated. Furthermore, the analysis and validation of the data took place through the use of multiple linear regression and other pertinent statistical techniques. The results indicate that endogenous and exogenous factors and the disclosure of SDGs influence the Market-to-Book of companies.

Keywords: disclosure, SDG, market to book, public companies 


\section{Influencia de factores exógenos y endógenos y divulgación de los ODS en el desempeńo de las empresas cotizadas}

A pesar del desarrollo de estudios sobre divulgación social y ambiental por parte de la comunidad científica, se ha avanzado poco en la incorporación de los Objetivos de Desarrollo Sostenible (ODS) como elementos de divulgación, ya sean factores endógenos (tamaño y nivel de gobierno corporativo) o exógenos (inversiones en carteras sostenibles e internacionalización) que afectan el nivel de divulgación de los ODS de manera diferente; o si existe una influencia directa e indirecta de estos factores en el desempeño. Desde esta perspectiva, esta investigación tiene como objetivo analizar si los factores exógenos y endógenos, y la divulgación relacionada con los ODS afectan el desempeño de las empresas cotizadas en la bolsa de valores de Brasil, Bolsa, Balcão - $[\mathrm{B}]^{3}$. Con este fin, se realizó una investigación cuantitativa y de carácter descriptivo, mediante la recopilación de datos documentales de informes de sostenibilidad, informes integrados y la base de datos Economatica ${ }^{\circledR}$ en el período de 2016 a 2018. A partir de los informes, se realizó un análisis de contenido; así, se obtuvo la cantidad de palabras asociadas a los ODS, a partir de las cuales se originó el índice de nivel de divulgación. Además, el análisis y la validación de los datos se llevó a cabo mediante el uso de regresión lineal múltiple y otras técnicas estadísticas pertinentes. Los resultados indican que factores endógenos y exógenos, y la divulgación de SAO influyen en el Market-to-Book de las empresas.

Palabras clave: evidenciación, ODS, market-to-book, empresas públicas

\section{Introdução}

O desenvolvimento sustentável é aquele que atende as demandas do presente, sem comprometer as necessidades das geraçōes futuras (World Commission on Environment and Development [WCED], 1987), sendo considerado de fundamental importância para o bem-estar social e a proteção do meio ambiente (United Nations, 2018). Em razão de sua relevância, se tornou um elemento central das açốes empreendidas pelas autoridades e instituições públicas, ao reconhecerem os benefícios e as fontes de desenvolvimento de longo prazo (Alinska, Filipiak, \& Kosztowniak, 2018).

Dessa forma, a interaçáo do desenvolvimento organizacional com a sustentabilidade se torna uma alternativa economicamente viável, ecologicamente correta e socialmente equitativa, baseada no princípio da eficiência na utilização dos recursos. Isso reforça o crescimento econômico e contribui com a geração de empregos, erradicação da pobreza e proteção ambien- tal (Programa das Naçóes Unidas para o Desenvolvimento [PNUD], 2019).

Os efeitos benéficos encorajaram o diálogo no meio empresarial sobre políticas de responsabilidade social corporativa (em diante, RSC) e açóes atreladas a sustentabilidade. Para incentivar açôes de sustentabilidade a Organização das Naçóes Unidas (em diante, ONU) estabeleceu o pacto global denominado de Objetivos de Desenvolvimento Sustentável (em diante, ODS), com a finalidade de engajar os governos, a sociedade e as empresas, aos problemas ambientais e sociais, visando a eficiência econômica, a equidade social e o equilíbrio ambiental (PNUD, 2019).

Apesar da filosofia do desenvolvimento sustentável ser reconhecida e pactuada pelas naçóes envolvidas com os ODS, colocá-los em prática requer esforço dos agentes públicos. Estimular as empresas a adotarem práticas sustentáveis requer comprometimento dos agentes públicos (Organização das Nações Unidas no Brasil [ONU 
Brasil], 2015). Os ODS são ousados pelos inúmeros desafios e conflitos de interesses associados a sua implementação, principalmente quando se busca a igualdade, equidade e justiça social e a proteção dos recursos naturais (Fleming, Wise, Hansen, \& Sams, 2017).

O alcance das metas da Agenda 2030 depende da forma como as empresas interpretam as oportunidades de crescimento, empreendedorismo e inovação trazidas pelo envolvimento com os ODS (World Business Council for Sustainable Development [WBCSD], 2021). Sendo assim, evidenciar as práticas desenvolvidas ganha relevância e as empresas têm reorientado as suas ações visando contribuir para a realização dos ODS. Isso decorre da compreensão de que o engajamento das empresas com os ODS melhora o nível de confiança dos stakeholders e incentiva comportamentos sustentáveis (Lecuona, Pérez, Martínez, Romero, \& Maldonado, 2017).

Nesta perspectiva, a divulgação de informaçóes de cunho social e ambiental é um meio para estabelecer legitimidade das empresas perante seus funcionários, clientes e sociedade. Tal evidenciação possibilita as empresas demonstrarem açóes que impactam no meio ambiente e na sociedade, incluindo informaçóes sobre o cumprimento legal, a definição de políticas, a proteção do meio ambiente, entre outros fatores (Said, Omar, \& Abdullah, 2013).

Assim, a divulgação de informaçóes sobre práticas de sustentabilidade permite que as empresas obtenham vantagens competitivas sustentáveis, assim como, diversos fatores exógenos ou endógenos podem impactar no nível de evidenciaçáo dos relatórios (Rosati \& Faria, 2019) e no desempenho das organizaçóes (Machado \& Machado, 2011).

A partir do contexto apresentado, surge a pergunta de pesquisa que orienta este estudo: qual a influência dos fatores exógenos e endógenos e da evidenciação relacionada aos ODS no desempenho de companhias abertas listadas na bolsa de valores Brasil, Bolsa, Balcáo $[\mathrm{B}]^{3}$ ? Diante do contexto, se apresenta como objetivo de pesquisa analisar se fatores exógenos e endógenos e a evidenciação relacionada aos ODS afetam o desempenho em companhias abertas listadas na bolsa de valores Brasil, Bolsa, Balcão $[\mathrm{B}]^{3}$.

Para responder a problemática de pesquisa e alcançar o objetivo proposto, utilizou-se a estratégia metodológica de pesquisa quantitativa, de caráter descritivo e por meio de coleta de dados documental em relatórios de sustentabilidade e banco de dados da Economatica $^{\circledR}$, no período de 2016 a 2018. A partir dos relatórios, foi realizada uma análise de conteúdo, obtendo o número de palavras associadas aos ODS, das quais se originou o índice do nível de evidenciação. A análise dos dados foi realizada a partir de dois conjuntos de dados, sendo utilizados para os testes de hipóteses e análise de sensibilidade das variáveis, respectivamente. A técnica de análise utilizada foi a regressão linear múltipla.

A relevância do estudo consiste no fato de as empresas buscarem, por meio de estruturas de divulgação, respostas às pressóes das partes interessadas, evidenciando em seus relatórios os atos e fatos de suas atividades que podem afetar o meio ambiente e a sociedade. A evidenciação social e ambiental, portanto, é considerada um diálogo entre as empresas e os seus stakeholders. As pressões políticas e sociais aumentam em função do menor desempenho ambiental e social das empresas, tornando à evidenciação uma estratégia de comunicação da capacidade da empresa (Meng, Zeng, Shi, Qi, \& Zhang, 2014).

Justifica-se ainda que, apesar do desenvolvimento de estudos sobre evidenciaçáo social e ambiental pela 
comunidade cientifica, pouco se tem avançado sobre a incorporação dos ODS como elementos de evidenciação. O que se espera é que as metas dos ODS sejam incorporadas pelas empresas e as informaçóes evidenciadas em seus relatórios, conforme estabelecido pela própria Agenda 2030: "incentivar as empresas, especialmente as empresas grandes e transnacionais, a adotar práticas sustentáveis e a integrar informaçóes de sustentabilidade em seu ciclo de relatórios" (ONU Brasil, 2015).

Por fim, a pesquisa proporciona contribuiçóes teóricas relacionadas a importância das empresas aderirem aos ODS para atingirem resultados econômicos satisfatórios e que determinados fatores da empresa e do seu ambiente podem também estimular a concretização da agenda global. A compreensão das características das empresas e do ambiente que promovem os ODS pode servir de base para que o poder público promova estratégias de engajamento das empresas desestimuladas. Além disso, o poder público pode elaborar projetos que ofereçam condiçôes para as empresas com estruturas interna deficitárias também consigam alcançar a consecução dos ODS e o desempenho desejado.

\section{Referencial teórico e hipóteses de pesquisa}

\subsection{Interface da evidenciaçáo dos ODS com o desempenho organizacional}

Muito se tem abordado a respeito da importância em tornar as organizaçóes conscientes de sua responsabilidade, por meio de uma mudança positiva em suas atitudes e mentalidade na realização de negócios, para que estes proporcionem melhoria do bem-estar e garantam simultaneamente, competitividade, crescimento organizacional e desenvolvimento sustentável (Hoque, Rahman, Molla, Noman, \& Bhuiyan, 2018). Todavia, alcançar o desenvolvimento sustentável tem sido desafiador e, muitas vezes, negligenciado pelo conceito da maximização de lucro, autocentrado e instalado como único e principal objetivo das organizaçóes empresariais (Hoque et al., 2018).

Com a adoção dos 17 ODS pelas ONU, as organizaçóes que priorizaram a maximização de lucro, passaram a dar maior importância aos demais stakeholders, não focalizando apenas no interesse dos acionistas (Hoque et al., 2018). Assim, as empresas têm reorientado suas açôes, visando contribuir para uma sociedade melhor e um ambiente seguro, priorizando clientes, funcionários, governo e sociedade.

Nesse sentido, a literatura tem demonstrado que a evidenciação social e ambiental das empresas pode ser uma ferramenta estratégica para atingir metas financeiras e atender as partes interessadas (Haninun, Lindrianasari, \& Denziana, 2018), sendo também um meio de empreender boa governança corporativa para melhorar as decisões e estratégias ambientais e sociais (Adinehzadeh, Jaffar, Shukor, \& Abdul Rahman, 2018), o cumprimento fiscal (Fallan \& Fallan, 2019), as previsões econômicas e o valor de mercado (Andrade, Salazar, Calegário, \& Silva, 2009).

A evidenciação é realizada por um conjunto de meios (relatórios de cunho social e ambiental) utilizados pelas empresas para demonstrar à sociedade como são gerenciados os impactos causados ao meio ambiente em decorrência de suas atividades (Rosa, Guesser, Hein, Pfitscher \& Lunkes, 2015). A conscientização criada pela evidenciação ambiental pressiona as empresas para que forneçam informaçóes relacionadas às atividades desenvolvidas. Desta forma, se nota uma tendência de as empresas divulgarem informaçôes em relatórios específicos para proporcionar maior transparência nas questóes ambientais, sociais e de governança. 
Nessa perspectiva, diferentes fatores da organização afetam o nível de evidenciação, sejam estes fatores endógenos ou exógenos (Rosati \& Faria, 2019). Todavia, embora, muitos estudiosos tenham analisado a influência de ambos fatores (internos e externos) sobre os relatórios de sustentabilidade, não há concordância sobre qual dimensão é dominante. Portanto, é provável que ambas as dimensóes possam desempenhar um papel significativo (Rosati \& Faria, 2019).

Por conseguinte, estudos apontam impacto positivo da responsabilidade social no desempenho das empresas (Machado \& Machado, 2011). Dessa forma, é de fundamental relevância compreender como fatores associados ao tamanho, governança corporativa, carteiras de investimento socialmente responsável e empresas internacionalizadas, por exemplo, podem afetar o desempenho de mercado, mensurado pelo Market-to-Book. O Market-to-Book é uma das medidas mais utilizadas no mercado de capitais para evidenciar as oportunidades de crescimento e expectativas futuras dos diversos agentes em relaçáo a empresa, ou seja. o quanto a empresa é valorizada pelo mercado em relação ao seu valor contábil (Almeida, Lopes \& Corrar, 2013).

Rosati e Faria (2019) indicam que a adoção dos ODS nos relatórios está associada diretamente as grandes empresas com níveis mais elevados de comprometimento com a estrutura de sustentabilidade e seu impacto externo. Como apontam Gamerschlag, Möller e Verbeeten (2011), grandes empresas são mais visíveis ao público e estão sujeitas a maiores pressóes políticas e regulamentares, dessa forma, ao divulgar mais informaçóes acabam por demonstrar que suas ações são legítimas.

Adicionalmente, Andrade et al. (2009), e Han, Zhuangxiong e Jie (2018) argumentam que as grandes empresas por já estarem consolidadas no mercado, acabam tendo melhores expectativas dos investidores na valorização das ações e, consequentemente, impactos positivos no desempenho. Logo, se apresenta como hipótese:

H1a: o tamanho da empresa influencia positivamente o desempenho de mercado.

Outro aspecto que influencia o desempenho é a governança corporativa, que passou a ser uma das principais credenciais para os negócios, devido ao fato de ser um elemento estratégico capaz de auxiliar no incremento dos lucros e das oportunidades de desenvolvimento (Macedo \& Corrar, 2012). Assim, a adoção de melhores práticas de governança corporativa acaba por afetar o desempenho das organizaçóes e sua interação junto ao mercado de capitais, sendo os níveis de governança uma alternativa de avaliação. Implantados em dezembro de 2000 pela então Bolsa de Valores de São Paulo (Bovespa), hoje Brasil, Bolsa, Balcão, o Novo Mercado e os níveis diferenciados de governança corporativa (níveis 1 e 2) são segmentos especiais de listagem, desenvolvidos com o objetivo de proporcionar um ambiente de negociação que estimule o interesse dos investidores, a valorização das companhias, que minimize os conflitos de agência e sirva de referência para a tomada de decisão (Vieira, Velasquez, Losekann, \& Ceretta, 2011; Vilhena \& Camargos, 2015; Caixe \& Krauter, 2014). Diante de tal contexto, se apresenta a seguinte hipótese de pesquisa:

H1b: níveis diferenciados de governança corporativa influenciam positivamente o desempenho de mercado.

Por sua vez, mercados globalizados e dinâmicos, com alto nível de competitividade, consumidores mais exigentes e conscientes, ocasiona a busca de elementos que diferenciem as empresas de seus concorrentes (Machado \& Machado, 2011). Logo, baseado em uma 
forte tendência mundial de valorização das empresas socialmente responsáveis, surge no Brasil, em 2005, o Índice de Sustentabilidade Empresarial (em diante, ISE), um índice de açôes considerado referencial para os investimentos socialmente responsáveis, capaz de fortalecer o mercado de capitais. Investidores que buscam as empresas integrantes do ISE para aplicar seus recursos, consideram que estas empresas "sustentáveis” são capazes de gerar maior valor para o acionista no longo prazo, pois estáo mais bem preparadas para enfrentar riscos econômicos, sociais e ambientais (Souza, Rásia, \& Jacques, 2010). A partir do exposto, emerge a hipótese:

H2a: empresas que compóe carteiras de investimento socialmente responsável apresentam maiores níveis de desempenho de mercado.

Ao se discutir sobre evidenciação ambiental, cabe salientar que muitas empresas têm avançado na implementação de sistemas de gestão de sustentabilidade (Martinuzzi \& Krumay, 2013). Associado a isso, tem-se a adesão aos padróes internacionais de relatórios de responsabilidade e sustentabilidade que se tornaram uma prática comum, o que melhora a transparência das atividades desenvolvidas e o impacto na cadeia de valor (Schönherr, Findler \& Martinuzzi, 2017).

Além disso, as empresas que atuam em nível internacional estão em maior escrutínio público, principalmente no que se refere aos ODS, que envolvem um pacto global. As empresas com atuação global tendem a receber maior cobrança regulatória, da sociedade e dos órgãos internacionais para aderirem ao pacto global. Diante disso, a percepção dos stakeholders pode afetar positivamente o desempenho das empresas internacionalizadas, ao aumentar sua lucratividade e valor aos acionistas (Schönherr et al., 2017). Nesse sentido, se propõe como hipótese:
H2b: empresas com internacionalização apresentam maiores níveis de desempenho de mercado.

Estudos de Chijoke-Mgbame e Mgbame (2018), Xia, Olanipekun, Chen, Xie e Liu (2018) e Fleming et al. (2017) constataram que as organizaçóes que evidenciam informaçôes voluntárias melhoraram a imagem corporativa e ganham vantagem competitiva. Percebe-se que as ações de sustentabilidade evidenciadas nos relatórios voluntários pode ser fator preponderante para a imagem corporativa que se reflete em resultados econômicos favoráveis. Com isso, é relevante que os negócios possuam RSC pelo engajamento com os ODS para equilibrar o desempenho social, ambiental e financeiro (Hoque et al., 2018).

As evidências empíricas sugerem a existência de relação amplamente positiva entre o desempenho em RSC e o desempenho econômico do empreendimento (Hoque et al., 2018). A RSC desempenha um papel importante na obtenção de vantagem competitiva, aprimorando a reputaçáo corporativa e aumentando o valor da empresa (Han et al., 2018). A manutençáo do valor de mercado exige alinhamento com as expectativas de mercado, a fim de sinalizar para acionistas e potenciais investidores uma boa reputação da firma, o que pode aumentar a liquidez das açóes e o valor da companhia (Almeida et al., 2013). Isso pode ser obtido pelas açóes de RSC que estão atreladas a adesão aos ODS. A partir disso, se tem como hipótese:

H3: o nível de evidenciação dos ODS afeta positivamente o desempenho de mercado.

A RSC e a gestão sustentável geram interferências no desempenho dos negócios (Xia et al., 2018). Sob está ótica, os relatórios sociais e ambientais estariam intrinsecamente relacionados ao desempenho financeiro. De acordo com a Teoria da Legitimidade, as empresas procuram legitimar suas atividades socioambientais 
junto às expectativas da sociedade, minimizando as preocupações sociais e obtendo vantagem competitiva que decorre da imagem corporativa (Suttipun \& Stanton, 2012).

A adoção de boas práticas de governança corporativa associada à evidenciação de informaçóes socioambientais permite melhorar a imagem corporativa junto ao mercado, que na sua extensão melhora os níveis de desempenho organizacional (Vilhena \& Camargos, 2015). Nesta perspectiva, tais empresas devem ser mais bem avaliadas pelo mercado do que aquelas que não as adotam. Diante de tal perspectiva, se apresenta a seguinte hipótese:

H4: o nível de evidenciação dos ODS modera positivamente a relação entre os níveis diferenciados de governança corporativa e o desempenho de mercado.

Os ODS são frequentemente percebidos pelas empresas transnacionais como uma oportunidade para reviver o crescimento e a inovação tecnológica, criando ganho para a sociedade e ao meio ambiente (Schönherr et al., 2017). Além do argumento comercial para o envolvimento das empresas multinacionais com os ODS, os objetivos globais adicionam oportunidades no enfrentamento dos desafios sistêmicos que atentam às expectativas da sociedade, contribuindo para o desenvolvimento sustentável na cadeia de valor (Schönherr et al., 2017).

Nos países que valorizam mais as necessidades sociais e ambientais, as empresas podem sofrer maior pressão para engajar-se nestas questóes, tanto do público quanto do sistema jurídico, podendo refletir na sustentabilidade e no desempenho (Rosati \& Faria, 2019). Diante do contexto apresentado, se propõe como hipótese:

H5: o nível de evidenciação dos ODS modera positivamente a relação de internacionalização das empresas com o desempenho de mercado.

Assim, partindo dos pressupostos teóricos de que a evidenciação, os fatores endógenos e exógenos afetam o desempenho, e que a evidenciação modera o efeito desses fatores e, consequentemente, o desempenho das empresas, o modelo teórico da pesquisa está representado na figura 1 .

\section{Figura 1. Modelo conceitual}

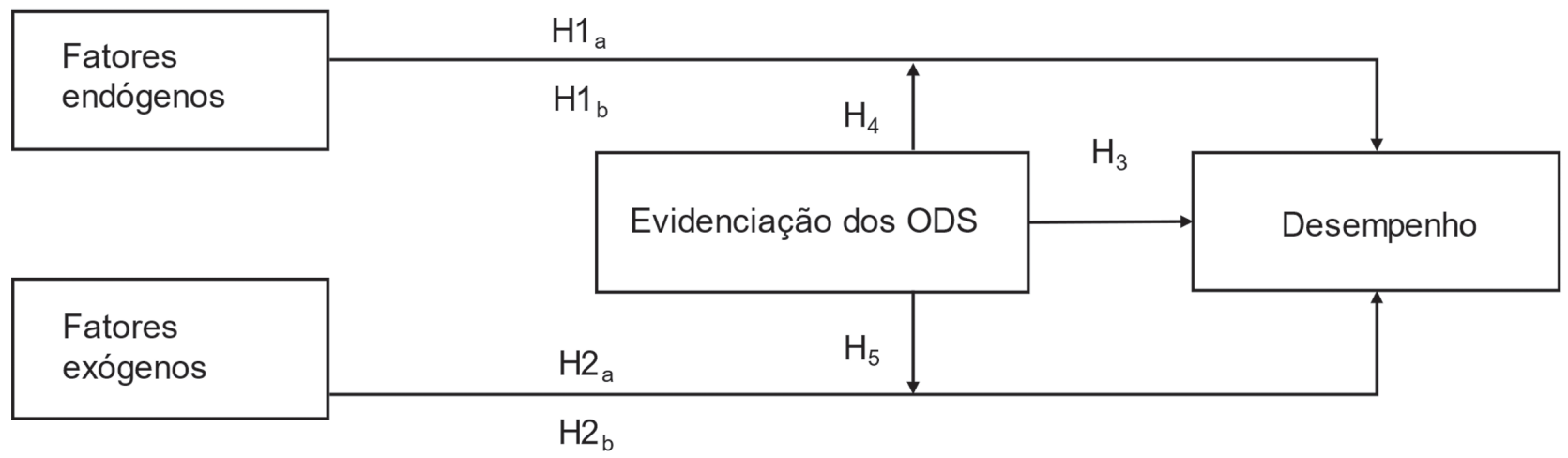

Fonte: Dados da pesquisa 
A estruturação do modelo conceitual representa as hipóteses de pesquisa, as quais foram desenvolvidas a partir do entendimento de que os fatores endógenos e os fatores exógenos afetam o desempenho. Já o nível de evidenciação dos ODS (em diante, ID_ODS) influencia diretamente o desempenho e modera a influência dos fatores endógenos e os fatores exógenos sobre o desempenho.

\section{Procedimentos metodológicos}

A pesquisa caracteriza-se como descritiva, conduzida por coleta documental (relatórios e base de dados) e com abordagem predominantemente quantitativa. A população da pesquisa está relacionada com as companhias abertas listadas na bolsa de valores brasileira, denominada Brasil, Bolsa, Balcão $[\mathrm{B}]^{3}$. Para a definição da amostra investigada foram excluídas as empresas do setor financeiro, em razão das peculiaridades da atividade econômica desenvolvida, e aquelas que não possuíam informaçóes necessárias ou completas dos anos em análise para operacionalizar as variáveis.

Considerando que o marco normativo dos ODS é o ano de 2015, a coleta documental utilizou-se dos relatórios de sustentabilidade para análise de conteúdo relativos aos exercícios de 2016 a 2018. A fim de verificar o nível de ID_ODS pelas empresas, a mensuração deu-se pela busca e apreciação de 60 palavras-chaves e expressóes relacionadas aos ODS divulgados nos relatórios analisados, utilizando-se da análise léxica (Gamerschlag et al., 2011).

Tabela 1. Expressóes utilizadas para o cálcullo do índice da ID_ODS

\begin{tabular}{|l|l|l|}
\hline \multicolumn{1}{|c|}{ Agricultura sustentável } & \multicolumn{1}{|c|}{ Indústria } & \multicolumn{1}{c|}{ ODS 13 } \\
\hline Água potável & Infraestrutura & ODS 14 \\
Aprendizagem & Inovaçáo & ODS 15 \\
Bem-estar & Instituiçáo Eficaz & ODS 16 \\
Cidade sustentável & Justiça & ODS 17 \\
Comunidade & Mudança Climática & ONU \\
Consumo responsável & Objetivos de Desenvolvimento Sustentável & ONU \\
Crescimento econômico & ODS & Parceria global \\
Desenvolvimento sustentável & ODS 1 & Parceria \\
Desertificação & ODS 2 & Paz \\
Desigualdade & ODS 3 & Pobreza \\
Ecossistema terrestre & ODS 4 & Produção responsável \\
Educaçáo & ODS 5 & Recurso marinho \\
Empoderamento feminino & ODS 6 & Saneamento \\
Empoderamento da mulher & ODS 7 & Saúde \\
Emprego & ODS 8 & Trabalho decente \\
Energia limpa & ODS 9 & Vida aquática \\
Energia renovável & ODS 10 & Vida na Água \\
Fome & ODS 11 & Vida saudável \\
Igualdade de gênero & ODS 12 & Vida terrestre \\
\hline
\end{tabular}

Fonte: Schio et al. (2019, p. 7). 
A escolha das palavras e expressóes (tabela 1) para coleta nos relatórios analisados foi amparada na pesquisa de Schio, Mazzioni, Moura e Dal Magro (2019), a qual embasou-se nos 17 ODS estabelecidos pela ONU. Cabe salientar, que quando aceitável, foi empregada a forma plural da expressão para minimizar possíveis inconsistências na coleta.

Para a busca e quantificação das expressóes, fez-se uso do software FineCount, o qual permitiu tabular e estratificar os termos definidos por análise de conteúdo. Ademais, foi adotado o número de palavras para construção do índice, conforme aplicado por Schio et al. (2019) e calculado de acordo com a equação 1.

$$
\text { ID_ODS }=\frac{\begin{array}{c}
\text { No de expressóes } \\
\text { relativas aos ODS }
\end{array}}{\begin{array}{c}
\text { No total de palavras } \\
\text { do relatório }
\end{array}}
$$

No intuito de proporcionar amplitude na análise, a amostra foi separada em dois grupos de empresas. O primeiro grupo foi constituído pelas 914 empresas que apresentaram todas as variáveis explicativas. Neste caso, para as empresas que não publicaram o relatório de sustentabilidade atribuiu-se escore 0 (zero) como índice de evidenciação. O segundo grupo foi constituído somente pelas 280 empresas que publicaram relatórios de sustentabilidade nos períodos analisados, permitindo calcular o índice de ID_ODS (Eq. 1). A utilização do grupo de 914 empresas permite testar as hipóteses de pesquisa, e por conseguinte o grupo composto por 280 empresas é utilizado para os testes das inferências estatísticas do modelo proposto, ao considerar apenas empresas que divulgaram relatórios. Para identificar os fatores endógenos e exógenos da ID_ODS nos relatórios, foram utilizadas as variáveis

\begin{tabular}{|c|c|c|c|c|}
\hline \multicolumn{2}{|c|}{ Variáveis independentes } & Métrica & Autores & Fonte \\
\hline \multirow{4}{*}{$\begin{array}{l}\text { Fatores } \\
\text { endógenos e } \\
\text { exógenos }\end{array}$} & Tamanho & $\begin{array}{l}\text { Logaritmo natural do valor contábil do ativo } \\
\text { total no final de cada período. }\end{array}$ & $\begin{array}{l}\text { Ashfaq Zhang, Munaim, Razzaq } \\
\text { (2016). }\end{array}$ & \multirow[b]{2}{*}{ Economatica } \\
\hline & $\begin{array}{l}\text { Governança } \\
\text { corporativa }\end{array}$ & $\begin{array}{l}\text { Variável dicotômica, sendo } 1 \text { para empresas } \\
\text { pertencentes a nível de governança (em diante, } \\
\text { NGC) diferenciados e } 0 \text { para as demais. }\end{array}$ & $\begin{array}{l}\text { Vilhena e Camargos (2015), Caixe } \\
\text { e Krauter (2014), Rosa et al. } \\
\text { (2015). }\end{array}$ & \\
\hline & $\begin{array}{l}\text { Investimento } \\
\text { socialmente } \\
\text { responsável }\end{array}$ & $\begin{array}{l}\text { Variável dicotômica, sendo } 1 \text { para empresas } \\
\text { pertencentes a carteira ISE e } 0 \text { para as demais. }\end{array}$ & $\begin{array}{l}\text { Almeida-Santos, Dani, Krespi e } \\
\text { Lavarda (2013), Rosa et al. (2015). }\end{array}$ & {$[\mathrm{B}]^{3}$} \\
\hline & $\begin{array}{l}\text { Internacionali- } \\
\text { zaçáa* }\end{array}$ & $\begin{array}{l}\text { Variável dicotômica, sendo } 1 \text { para empresas } \\
\text { com receitas relevantes oriundas do exterior e } \\
0 \text { para as demais. }\end{array}$ & Gondrige (2010). & {$[\mathrm{B}]^{3}$} \\
\hline \multicolumn{2}{|c|}{ Nível de evidenciação de ODS } & $\begin{array}{l}\text { Expressóes utilizadas no relatório (tabela } 1 \text { e } \\
\text { equaçáo 1) }\end{array}$ & $\begin{array}{l}\text { ONU Brasil (2015), Lecuona et al. } \\
\text { (2017), Schio et al. (2019) }\end{array}$ & Websites \\
\hline \multicolumn{2}{|c|}{ Variáveis dependentes } & Métrica & Autores & Fonte \\
\hline $\begin{array}{l}\text { Desempenho } \\
\text { de Mercado }\end{array}$ & Market-to-Book & Valor de mercado das açóes/Patrimônio líquido & $\begin{array}{l}\text { Almeida et al. (2013), Vilhena e } \\
\text { Camargos (2015) }\end{array}$ & Economatica \\
\hline
\end{tabular}
descritas apresentadas na tabela 2, que contemplam as métricas e autores que já utilizaram tais variáveis.

Tabela 2. Operacionalização das variáveis

*Receitas relevantes oriundas do exterior são aquelas consideras pelas empresas no item 7.6 - Receitas relevantes no exterior, no Formulário de Referências. Fonte: Dados da pesquisa. 
A análise de dados ocorreu pelo uso das técnicas da estatística univariada, bivariada e multivaridada. Foram utilizados os seguintes modelos para as regressóes:

MKT $=\alpha 0+\alpha 1$ IE_ODS $+\alpha 2 \operatorname{LnTam}+\alpha 3 \operatorname{LnTam}+$ $\alpha 4$ INTER $+\alpha 5$ ISE $+\varepsilon$

(Equação 2)

MKT $=\alpha 0+\alpha 1 \mathrm{IE} \_O D S+\alpha 2 \operatorname{LnTam}+\alpha 3 \operatorname{LnTam}$ $+\alpha$ INTER + $\alpha 5$ ISE + $\alpha 6$ IE_ODS*NCG + $\alpha 6$ IE_ ODS*INTER $+\varepsilon$

\section{(Equação 3)}

Para validar os resultados da regressão linear múltipla, foram observados os pressupostos de normalidade, em que foi assumido o pressuposto do teorema do limite central (TLC) devido ao tamanho da amostra. Ademais, se fez uso de testes multicolinearidade, por meio do fator de inflação de variância - VIF e Tolerance; homocedasticidade, por meio do teste de PerasánPerasán, correção de heterocedasticidade pelo teste White; e ausência de autocorrelação serial, por meio do teste de Durbin-Watson.

\section{Discussóes e análises de dados}

A partir do índice de palavras, pode-se identificar as empresas que possuem maiores níveis de divulgação de informaçóes associadas aos ODS em seus relatórios, conforme apresentado na tabela 3 .

A tabela 3 apresenta as organizaçôes que compóe o ranking das 10 empresas com maiores índices de palavras para os anos de 2016 a 2018. Verifica-se que a BRF lidera o ranking do ano de 2016, a Aliansce Shopping em 2017 e a CPFL em 2018, respectivamente, com maior índice de palavras que fazem menção aos ODS. É possível observar que ocorreram alteraçóes das empresas que compóem a liderança, o que pode ser justificado pelo fato de que nem todas as organizaçôes publicaram seus relatórios para todos os anos analisados, dentro do período de coleta dos dados.

A tabela 4 demonstra a quantidade de citaçóes de cada um dos 17 ODS nos relatórios de sustentabilidade ou relatos integrados das empresas investigadas, no período de 2016 a 2018. Por meio dos resultados,

Tabela 3. Ranking das 10 empresas anuais com maior índice de menção aos ODS

\begin{tabular}{|c|c|c|c|c|c|}
\hline \multicolumn{2}{|c|}{ ANO DE 2016} & \multicolumn{2}{|c|}{ ANO DE 2017} & \multicolumn{2}{|c|}{ ANO DE 2018} \\
\hline EMPRESAS & ID Palavras & EMPRESAS & ID Palavras & EMPRESAS & ID Palavras \\
\hline 1. BRF & 0,105 & ALIANSCE SHOPPING & 0,03307 & CPFL & 0,0829 \\
\hline 2. NATURA & 0,071 & SANTOS BRASIL & 0,01913 & COSAN S.A. & 0,0262 \\
\hline 3. M.DIAS BRANCO & 0,052 & KLABIN - S.A. & 0,01843 & COSAN LOGISTICA & 0,0262 \\
\hline 4. KROTON EDU. & 0,036 & ECORODOVIAS & 0,01631 & BRASKEM S.A. & 0,0259 \\
\hline 5. COPEL & 0,024 & MRV ENGENHARIA & 0,01619 & EMBRAER & 0,0236 \\
\hline 6. BRASKEM & 0,020 & NATURA & 0,01485 & COPASA MG & 0,0181 \\
\hline 7. ENERGISA MG & 0,016 & TIM PARTICIPACOES & 0,01440 & CTEEP & 0,0155 \\
\hline 8. SUZANO & 0,015 & EDP & 0,01390 & ECORODOVIAS & 0,0151 \\
\hline 9. MULTIPLAN & 0,015 & WHIRLPOOL S.A. & 0,01315 & SAO MARTINHO S.A. & 0,0138 \\
\hline 10.CEMIG & 0,014 & AMPLA S.A. & 0,01279 & TELEFÔNICA BRASIL & 0,0132 \\
\hline
\end{tabular}

Fonte: Dados da pesquisa. 
é possível inferir que o ano de 2017 demonstrou maior nível de menção aos ODS, atestando uma evolução da abordagem do tema nos relatos das companhias. Por sua vez, o ano de 2018 também representou maior evidenciação em relação ao ano de 2016, contudo menor que 2017.

Tabela 4. Nível de ID_ODS nos relatórios no período de 2016 a 2018

\begin{tabular}{|c|c|c|c|}
\hline & 2016 & 2017 & 2018 \\
\hline ODS 1 & 80 & 164 & 42 \\
ODS 2 & 48 & 103 & 35 \\
ODS 3 & 94 & 218 & 137 \\
ODS 4 & 48 & 111 & 84 \\
ODS 5 & 106 & 246 & 116 \\
ODS 6 & 82 & 195 & 102 \\
ODS 7 & 111 & 252 & 133 \\
ODS 8 & 237 & 609 & 292 \\
ODS 9 & 70 & 155 & 93 \\
ODS 10 & 73 & 130 & 96 \\
ODS 11 & 90 & 143 & 101 \\
ODS 12 & 222 & 393 & 234 \\
ODS 13 & 136 & 269 & 137 \\
ODS 14 & 72 & 196 & 81 \\
ODS 15 & 109 & 213 & 109 \\
ODS 16 & 179 & 426 & 188 \\
ODS 17 & 54 & 125 & 67 \\
\hline Total & 1811 & 3948 & 2047 \\
\hline
\end{tabular}

Fonte: Dados da pesquisa.

Além disso, se pode constatar por meio de análise dos relatórios, e conforme resultados apresentados na tabela 4, que os ODS com maior menção nos anos analisados (2016 a 2018) foram: 12 - Consumo e produção responsável; 8 - Trabalho decente e crescimento econômico; 16 - Paz, justiça e instituiçóes eficazes. Diante disso, se verifica a priorização de aspectos atrelados ao desenvolvimento de organizações eficazes, responsáveis, transparentes, com uma gestão eficiente dos recursos e com um processo decisório responsivo e inclusivo em todos os níveis. Outro aspecto observado é o aumento no número de empresas que mencionou os ODS em seus relatórios, sendo 84 em 2016, 95 em 2017 e 101 em 2018. Na tabela 5 apresenta-se a correlaçáo de Pearson das variáveis explicativas.

Tabela 5. Correlaçáo das variáveis explicativas

\begin{tabular}{|l|c|c|c|c|c|}
\hline & IE_ODS & TAM & INTER & ISE & NGC \\
\hline ID_ODS & 1 & & & & \\
TAM & $0,278^{* *}$ & 1 & & & \\
INTER & $0,368^{* *}$ & $0,279^{* *}$ & 1 & & \\
ISE & $0,328^{* *}$ & $0,260^{* *}$ & $0,153^{* *}$ & 1 & \\
NGC & $0,114^{* *}$ & $0,415^{* *}$ & $0,174^{* *}$ & $0,222^{* *}$ & 1 \\
\hline
\end{tabular}

**. A correlação é significativa no nível 0,01 (2 extremidades).

Legenda: ID_OS (Evidenciação dos ODS); TAM (Tamanho); INTER (Internacionalização); ISE; NGC.

Fonte: Dados da pesquisa.

Os resultados da tabela 5 indicam que as relaçôes entre as variáveis foram todas positivas e estatisticamente significativas, variando entre 0,114 e 0,415 . Os escores são considerados fracos $(0,10$ até 0,30$)$ e moderados $(0,40$ até 0,6$)$. Os resultados da correlação de Pearson indicam que não existem problemas de multicolinearidade entre as variáveis preditoras, podendo-se utilizar de modo conjunto no modelo de regressão linear múltipla.

Com base no modelo conceitual e das hipóteses de pesquisas, procedeu-se a análise por meio da regressão linear múltipla. Inicialmente, foram testadas as hipóteses utilizando o grupo de 914 empresas, sendo que destas 280 publicaram relatórios de sustentabilidade ou integrado, cujos resultados podem ser observados na tabela 6 . 
Tabela 6. Resultados da regressáo no período de 2016 a 2018

\begin{tabular}{|c|c|c|c|c|}
\hline \multirow{3}{*}{$\begin{array}{l}\text { Variável Dependente } \\
\text { Variáveis explicativas }\end{array}$} & \multicolumn{4}{|c|}{ Market-to-Book } \\
\hline & \multicolumn{2}{|c|}{ Equação 2} & \multicolumn{2}{|c|}{ Equaçáo 3} \\
\hline & Coef. B & Estat. T & Coef. B & Estat. T \\
\hline Constante & $-0,367$ & $-0,820$ & $-0,450$ & $-1,010$ \\
\hline ID_ODS & 22,970 & $1,65^{*}$ & $-8,280$ & 0,710 \\
\hline NGC & 0,815 & $5,59^{* * *}$ & 0,719 & $4,690^{* * *}$ \\
\hline LN_Tam & 0,075 & $2,40^{* * *}$ & 0,085 & $2,750^{* * *}$ \\
\hline INTER & 0,438 & $1,680^{*}$ & 0,410 & 1,570 \\
\hline ISE & 1,254 & $3,590^{* * *}$ & 0,883 & $2,200^{* *}$ \\
\hline ID_ODS*NCG & - & - & 41,778 & $2,030^{* *}$ \\
\hline ID_ODS*INTER & - & - & 37,113 & $1,780^{*}$ \\
\hline Estatística F & \multicolumn{2}{|c|}{$22,640^{* * *}$} & \multicolumn{2}{|c|}{$20,660^{* * *}$} \\
\hline $\mathrm{R}^{2}$ ajustado & \multicolumn{2}{|c|}{0,136} & \multicolumn{2}{|c|}{0,143} \\
\hline Tolerance & \multicolumn{2}{|c|}{1 a 0,10} & \multicolumn{2}{|c|}{1 a 0,10} \\
\hline VIF & \multicolumn{2}{|c|}{1,183 a 1,297} & \multicolumn{2}{|c|}{1,376 a 3,332} \\
\hline DW & \multicolumn{2}{|c|}{2,020} & \multicolumn{2}{|c|}{2,015} \\
\hline Pesarán-Pesarán (sig) & \multicolumn{2}{|c|}{0,043} & \multicolumn{2}{|c|}{0,355} \\
\hline $\mathrm{N}$ & \multicolumn{2}{|c|}{914} & \multicolumn{2}{|c|}{914} \\
\hline \multicolumn{5}{|c|}{ Significativo ao nível de $10 \%^{*}, 5 \%^{* *}, 1 \%^{* * *}$} \\
\hline Correção de heterocec & & & & \\
\hline
\end{tabular}

Legenda: ID_OS; TAM (Tamanho); INTER (Internacionalização); ISE; NGC.

Fonte: Dados da pesquisa.

Nota-se na tabela 6, que os fatores endógenos, exógenos e a ID_ODS (Equação 2), explicam, conjuntamente, cerca de $13,6 \%$ ( $\mathrm{R}^{2}$ ajustado do modelo) do desempenho de mercado das empresas. Ao se acrescentar a moderaçáo da evidenciação com o NGC corporativa e internacionalização (Equação 3), o $\mathrm{R}^{2}$ ajustado aumentou para $14,3 \%$ e as variáveis explicativas apresentaram relaçáo positiva com o desempenho de mercado, ao nível de 1\% (estatística F).

Em relação aos fatores endógenos (NGC e tamanho), exógenos (internacionalização e ISE) e nível de ID_

ODS, verifica-se que todas as variáveis mostraram significância, influenciando no aumento do desempenho de mercado das organizaçóes (Equação 2). O resultado está em linha com o preconizado pela teoria e acarreta na confirmação das hipóteses de pesquisa $\mathrm{H} 1 \mathrm{a}, \mathrm{H} 1 \mathrm{~b}$, $\mathrm{H} 2 \mathrm{a}, \mathrm{H} 2 \mathrm{~b}$ e H3.

Dentre as variáveis endógenas, percebe-se que NGC apresentou coeficiente positivo e estatisticamente significativo ao nível de $1 \%$ com a variável dependente (desempenho) em ambos os modelos, o que demonstra que a governança corporativa pode ser considerada como elemento estratégico (Macedo \& Corrar, 2012), que beneficia as organizaçóes na obtenção de valorização junto ao mercado de capitais (Vieira et al., 2011).

Os resultados não permitem rejeitar a hipótese $\mathrm{H} 1 \mathrm{~b}$. Estes resultados corroboram com os achados de Caixe e Krauter (2014) e Vilhena e Camargos (2015), os quais apontaram que empresas com adesão aos níveis diferenciados de governança corporativa da $[\mathrm{B}]^{3}$ apresentaram maior valor de mercado, indicando que os investidores pagam valores adicionais dos ativos de tais empresas em comparação às demais, o que resulta em melhor valorização das açôes.

A variável tamanho se mostrou relacionada positivamente e significativa ao nível de $1 \%$ com o Market-to-Book. Empresas de maior porte e com maior visibilidade estão mais propensas à divulgação de informaçóes econômicas, ambientais e sociais, o que proporciona valorização e legitimidade junto ao mercado de capitais (Rosati \& Faria, 2019; Gamerschlag et al., 2011). Estes resultados validam a hipótese H1a e apoiam os achados de Vilhena e Camargos (2015), e Han et al. (2018), ao demonstrar que quanto maior o tamanho da empresa maior o desempenho de mercado. 
Quanto a variável de nível de evidenciação (ID_ODS) se percebe que o modelo da Equação 2, apresentou nível de significância estatística de $10 \%$ com a variável dependente, o que demonstra que empresas com maior nível de evidenciação de informaçóes sobre os ODS são mais reconhecidas pelo mercado de capitais. Estes resultados coadunam os achados de Chijoke-Mgbame e Mgbame (2018), Xia et al. (2018) e Fleming et al. (2017), ao identificar que as organizaçóes ao evidenciar voluntariamente suas informações obtiveram impacto positivo no desempenho. Os achados não permitem rejeitar a hipótese $\mathrm{H} 3$, de que o nível de ID_ODS afeta positivamente o desempenho.

Ressalta-se que o efeito moderador da evidenciação (ID_ODS) na relação entre a governança corporativa e o desempenho, se mostrou significativo, não rejeitando-se a hipótese H4. Este resultado indica que a ID_ODS melhora o efeito da governança corporativa no desempenho de mercado das firmas. Resultado parecido pode ser obtido no efeito moderador da evidenciação (ID_ODS) na relação entre internacionalização e o desempenho de mercado, não podendo ser rejeitada a hipótese H5. Confirma-se que a ID_ODS faz emergir um melhor posicionamento da interferência positiva da internacionalização no desempenho de mercado das firmas.

Compete revelar ainda que dentre as empresas que realizam a evidenciação dos seus relatórios de sustentabilidade (280 empresas), apenas 191 se encontram nos níveis diferenciados de governança corporativa (Nível 1, Nível 2 e Novo Mercado) da listagem Brasil, Bolsa, Balcão. E destas, apenas 96 organizaçóes possuem receitas relevantes oriundas do exterior (item 7.6 - Receitas relevantes no exterior, no Formulário de Referências).

Por sua vez, quanto as variáveis exógenas, é possível constatar que a variável ISE apresentou significância estatística e positiva com o desempenho ao nível de $1 \%$ e posteriormente ao nível de $5 \%$, respectivamente (Equação 2 e 3). A justificativa pode estar relacionada ao fato de que a sociedade passou a valorizar empresas socialmente responsáveis e os investidores buscam organizaçóes integrantes do ISE para aplicar seus recursos.

Assim, por meio dos resultados, não é possível rejeitar a hipótese $\mathrm{H} 2 \mathrm{a}$, de que as empresas que compóe carteiras de investimento socialmente responsável apresentam maiores níveis de desempenho. Além disso, corroboram os achados da pesquisa de Cristófalo, Akaki, Abe, Morano e Miraglia, (2016), de que empresas pertencentes ao ISE apresentaram melhor desempenho. Andrade et al. (2009) apontam que estes resultados podem ser atrelados ao tamanho da empresa e o nível de impacto ambiental inerente ao setor e as suas atividades, determinantes para a adesão ao ISE.

Ademais, ao examinar a tabela 6 é possível perceber que a variável INTER apresentou significância ao nível de $10 \%$ em relação ao desempenho (Equação 2), o que não permite rejeitar a hipótese $\mathrm{H} 2 \mathrm{~b}$. Este resultado indica um melhor desempenho de empresas internacionalizadas, como apontado por Gondrige (2010). Por conseguinte, ao observar o efeito moderador da ID_ODS com a internacionalização, pode-se assegurar uma inferência positiva ao nível de $10 \%$ no desempenho das empresas, o que valida a hipótese $\mathrm{H} 5$.

Os resultados podem ter sido influenciados pela quantidade de empresas internacionalizadas que realizaram a ID_ODS no período analisado, sendo que estas podem estar sujeitas ao maior escrutínio público e maior necessidade de legitimação, constituindo-se em fatores de maior exigência da evidenciação de informaçóes atreladas a RSC. Posteriormente, foi elaborada uma análise de sensibilidade utilizando-se da regres- 
são linear múltipla com a amostra composta apenas pelas empresas que publicaram os relatórios, conforme pode ser observado na tabela 7 .

Tabela 7. Resultados da regressão de empresas com ID_ODS no período de 2016 a 2018

\begin{tabular}{|c|c|c|c|c|}
\hline \multirow{3}{*}{$\begin{array}{l}\text { Variável Dependente } \\
\text { Variáveis explicativas }\end{array}$} & \multicolumn{4}{|c|}{ Market-to-Book } \\
\hline & \multicolumn{2}{|c|}{ Equação 2} & \multicolumn{2}{|c|}{ Equação 3} \\
\hline & Coef. B & Estat. T & Coef. B & Estat. T \\
\hline Constante & 1,142 & 0,812 & 2,090 & 1,490 \\
\hline ID_ODS & 26,291 & $2,121^{* *}$ & $-25,864$ & $-1,198$ \\
\hline NGC & 0,759 & $2,820^{* * *}$ & 0,122 & 0,712 \\
\hline LN_Ativo Total & $-0,020$ & $-0,233$ & $-0,053$ & $-0,613$ \\
\hline INTER & 0,540 & $2,053^{* *}$ & 0,212 & 0,639 \\
\hline ISE & 1,151 & $3,826^{* * *}$ & 1,083 & $3,669^{* * *}$ \\
\hline ID_ODS*NCG & - & - & 82,173 & $0,001^{* * *}$ \\
\hline ID_ODS*INTER & - & - & 37,177 & 0,139 \\
\hline Estatística F & \multicolumn{2}{|c|}{$8,780^{* * *}$} & \multicolumn{2}{|c|}{$8,466^{* * *}$} \\
\hline $\mathrm{R}^{2}$ ajustado & \multicolumn{2}{|c|}{0,122} & \multicolumn{2}{|c|}{0,179} \\
\hline Tolerance & \multicolumn{2}{|c|}{1 até 0,10} & \multicolumn{2}{|c|}{1 até 0,10} \\
\hline VIF & \multicolumn{2}{|c|}{1,041 a 1,125} & \multicolumn{2}{|c|}{1,054 a 3,823} \\
\hline DW & \multicolumn{2}{|c|}{2,021} & \multicolumn{2}{|c|}{1,994} \\
\hline Pesarán-Pesarán (sig) & \multicolumn{2}{|c|}{0,51} & \multicolumn{2}{|c|}{0,909} \\
\hline $\mathrm{N}$ & \multicolumn{2}{|c|}{280} & \multicolumn{2}{|c|}{280} \\
\hline
\end{tabular}

Legenda: ID_OS (Evidenciação dos ODS); TAM (Tamanho); INTER (Internacionalizaçâo); ISE; NGC (Nível de Governança).

Fonte: Dados da pesquisa.

Por meio dos dados evidenciados na tabela 7, é possível aferir que dentre as variáveis independentes na equação 2, NGC, INTER, ISE e ID_ODS apresentaram influência sobre o desempenho de mercado, enquanto a variável de tamanho não demonstrou significância estatística. Por sua vez, na equação com a inclusão das variáveis moderadoras, apenas a variável ISE manteve-se positivamente significativa e validando novamente a hipótese $\mathrm{H} 2 \mathrm{a}$.
Ademais, ao acrescentar ao modelo as variáveis moderadoras, tem-se ainda a aceitação da hipótese $\mathrm{H} 4$ - o nível de ID_ODS modera positivamente a relação entre os níveis diferenciados de governança corporativa e o desempenho, com nível de significância de $1 \%$. O resultado corrobora o pressuposto de que a responsabilidade socioambiental aumenta o desempenho, como exposto por Xia et al. (2018).

Sob está ótica, como apontam Suttipun e Stanton (2012), os relatórios sociais e ambientais estariam intrinsecamente relacionados ao desempenho financeiro, ao divulgar informaçóes a respeito de açóes ambientais, demonstrando que as empresas legitimam suas atividades frente às expectativas da sociedade, a fim de obter vantagem competitiva. A respeito da moderação das variáveis nível de evidenciação e internacionalização, referente a hipótese $\mathrm{H} 5$, verifica-se que o nível de ID_ODS não influencia significativamente a relação de internacionalização das empresas com o desempenho.

Logo, é possível constatar que quando as empresas que evidenciam os ODS são comparadas com seus pares, o nível de significância das variáveis em relação ao desempenho sofre alteraçóes. Por fim, constata-se que o mercado reconhece informaçóes que a contabilidade não consegue evidenciar, pela observância aos princípios contábeis ou dispositivos legais.

\section{Consideraçóes finais}

O estudo teve por objetivo analisar se fatores exógenos, endógenos e a evidenciação relacionada aos ODS afetam o desempenho em companhias abertas listadas na bolsa de valores Brasil, Bolsa, Balcão $[\mathrm{B}]^{3}$. Os resultados sugerem que fatores endógenos (tamanho e nível de governança), exógenos (investimentos socialmente responsáveis e internacionalização) e nível 
de ID_ODS impactam positivamente no desempenho de mercado das organizações, sugerindo que a divulgação de informações sociais e ambientais nos relatórios possibilita às empresas obterem vantagens competitivas em comparação às demais.

Todavia, constata-se a impossibilidade de assumir qual a dimensão (exógeno ou endógeno) é dominante na influência do desempenho de mercado, uma vez que ambas representam um papel significativo e possuem características distintas. Quando se tem a moderação das variáveis internacionalização e nível de governança com a ID_ODS, acaba por refletir alteraçóes sobre as variáveis que impactam no desempenho.

Referente a governança corporativa, os resultados indicam que a ID_ODS, conjuntamente, com a adoção de práticas de governança permite melhorar o desempenho das organizaçóes e sua imagem junto ao mercado, uma vez que perpassa aos stakeholders maior transparência e credibilidade, como apontado por Vilhena e Camargos (2015). Não obstante, cabe salientar que apesar da publicação de relatórios de sustentabilidade não ser obrigatória, verifica-se uma tendência global em que os investidores buscam empresas socialmente responsáveis, sustentáveis e rentáveis para aplicar seus recursos. Dessa forma, empresas pertencentes ao ISE possuem maior legitimidade junto aos investidores, em relação a eficiência econômica, ambiental, social e de governança corporativa. Logo, os resultados obtidos sustentam essas afirmativas e corroboram com pesquisas anteriores, como a de Almeida-Santos et al. (2013) e Cristófalo et al. (2016).

A partir dos resultados é possível frisar que, apesar de menos de 1/3 das empresas terem divulgado seus relatórios de sustentabilidade, percebe-se uma tendência à adesão da ID_ODS. Tal comportamento pode ser decorrente da importância percebida pela transparência, prestação de constas e gestáo dos seus impactos frente a sociedade e aos demais stakeholders, fator comprovado por metodologias que já consideram os ODS em seu framework.

Nesta perspectiva, o estudo oferece ao menos duas contribuições: (i) a confirmação de que boas práticas de governança corporativa, presença em carteiras de investimentos socialmente responsáveis e tamanho das empresas agem como influenciadores para desempenho de mercado superior; (ii) as empresas estáo aderindo progressivamente aos ODS e a consequente evidenciação em seus relatórios, influenciando no aumento do valor de mercado. Embora as perspectivas de análise, a pesquisa apresentou limitações pela coleta de dados, pois nem todas as empresas divulgaram seus relatórios para todos os anos analisados, no período em que se realizou a coleta, fato que decorre da periodicidade de divulgação e metodologias distintas adotadas na elaboração dos relatos.

Para pesquisas futuras, se sugere a adoção de um período mais longo para análise e com segregação de setores específicos. Adicionalmente, se pode avaliar a influência dos ODS em outros indicadores de desempenho econômico e financeiro. Outra perspectiva é avaliar a interrelação entre os ODS contemplados pelas empresas.

\section{Referências}

Adinehzadeh, R., Jaffar, R., Shukor, Z. A., \& Abdul Rahman, M. R. C. (2018). The mediating role of environmental performance on the relationship between corporate governance mechanisms and environmental disclosure. Asian Academy of Management Journal of Accounting \& Finance, 14(1), 153-183. https://doi. org/10.21315/aamjaf2018.14.1.7

Alinska, A., Filipiak, B. Z., \& Kosztowniak, A. (2018). The importance of the public sector in sustainable development in Poland. Sustainability, 10(9), 3278, 1-24. https://doi.org/10.3390/su10093278 
Almeida, J. E. F. D., Lopes, A. B., \& Corrar, L. J. (2013). Gerenciamento de resultados para sustentar a expectativa do mercado de capitais: impactos no índice Market-to-Book. ASAA-Advances in Scientific and Applied Accounting, 4(1), 44-62. https://doi.org/10.14392/ ASAA.2011040103

Almeida-Santos, P. S., Dani, A. C., Krespi, N. T., \& Lavarda, C. E. F. (2013). Desempenho econômico e a responsabilidade social corporativa: uma contribuição para a análise da relação destas variáveis no caso das maiores companhias abertas Brasileiras. Enfoque: Reflexão Contábil, 32(1), 15-27. https://doi.org/10.4025/enfoque.v32i1.16761

Andrade, L. P. D., Salazar, G. T., Calegário, C. L. L., \& Silva, S. S. (2009). Governança corporativa: uma análise da relação do conselho de administração com o valor de mercado e desempenho das empresas brasileiras. RAM, Revista de Administração Mackenzie, 10(4), 4-31. https://doi.org/10.1590/S1678-69712009000400002

Ashfaq, K., Zhang, R., Munaim, A., \& Razzaq, N. (2016). An investigation into the determinants of risk disclosure in Banks: Evidence from financial sector of Pakistan. International Journal of Economics and Financial Issues, 6(3), 1049-1058.

Caixe, D. F., \& Krauter, E. (2014). The relation between corporate governance and market value: mitigating endogeneity problems. Brazilian Business Review, 11(1), 90-110. https://doi.org/10.15728/bbr.2014.11.1.5

Chijoke-Mgbame, A. M., \& Mgbame, C. O. (2018). Discretionary environmental disclosures of corporations in Nigeria. International Journal of Disclosure and Governance, 15(4), 252-261. https://doi.org/10.1057/ s41310-018-0052-0

Cristófalo, R. G., Akaki, A. S., Abe, T. C., Morano, R. S., \& Miraglia, S. G. E. K. (2016). Sustentabilidade e o mercado financeiro: estudo do desempenho de empresas que compóem o índice de sustentabilidade empresarial (ISE). REGE-Revista de Gestäo, 23(4), 286-297. https://doi.org/10.1016/j.rege.2016.09.001
Fallan, E., \& Fallan, L. (2019). Corporate tax behaviour and environmental disclosure: Strategic trade-offs across elements of CSR? Scandinavian Journal of Management, 35(3), 101042, 1-15. https://doi.org/10.1016/j. scaman.2019.02.001

Fleming, A., Wise, R. M., Hansen, H., \& Sams, L. (2017). The sustainable development goals: A case study. Marine Policy, 86, 94-103. https://doi.org/10.1016/j. marpol.2017.09.019

Gamerschlag, R., Möller, K., \& Verbeeten, F. (2011). Determinants of voluntary CSR disclosure: empirical evidence from Germany. Review of Managerial Science, 5(2-3), 233-262. https://doi.org/10.1007/s11846010-0052-3

Gondrige, E. O. (2010). Fatores explicativos do disclosure voluntário das empresas brasileiras de capital aberto: um estudo sob a perspectiva da estrutura de governança corporativa e de propriedade. (Dissertação de mestrado, Setor de Ciências Sociais Aplicadas, Universidade Federal do Paraná. Curitiba, Brasil). Recuperado de https://acervodigital.ufpr.br/handle/1884/24934 [Consulta: 8 de novembro de 2019].

Han, W., Zhuangxiong, Y., \& Jie, L. (2018). Corporate social responsibility, product market competition, and product market performance. International Review of Economics \& Finance, 56, 75-91. https://doi. org/10.1016/j.iref.2018.03.019

Haninun, H., Lindrianasari, L., \& Denziana, A. (2018). The effect of environmental performance and disclosure on financial performance. International Journal of Trade and Global Markets, 11(1/2), 138-148. https:// doi.org/10.1504/IJTGM.2018.092471

Hoque, N., Rahman, A. R. A., Molla, R. I., Noman, A. H. M., \& Bhuiyan, M. Z. H. (2018). Is corporate social responsibility pursuing pristine business goals for sustainable development? Corporate Social Responsibility and Environmental Management, 25(6), 1130-1142. https://doi.org/10.1002/csr.1527

Contabilidad y Negocios (16) 32, 2021 / ISSN 1992-1896 
Lecuona, A., Pérez, M., Martínez, P. S., Romero, G., \& Maldonado, M. (2017). The contribution of cement companies to the achievement of sustainable development goals and its effect in building trust in their communities. European Scientific Journal, ESJ, 13(29), 358-376. https://doi.org/10.19044/esj.2017.v13n29p358

Macedo, M. A. S., \& Corrar, L. J. (2012). Análise comparativa do desempenho contábil-financeiro de empresas com boas práticas de governança corporativa no Brasil. Revista Contabilidade e Controladoria, 4(1), 42-61. https://doi.org/10.5380/rcc.v4i1.25258

Machado, M. A. V., \& Machado, M. R. (2011). Responsabilidade social impacta o desempenho financeiro das empresas? ASAA-Advances in Scientific and Applied Accounting, 4(1), 2-23. https://doi.org/10.14392/ ASAA.2011040101

Martinuzzi, A., \& Krumay, B. (2013). The good, the bad, and the successful-how corporate social responsibility leads to competitive advantage and organizational transformation. Journal of Change Management, 13(4), 424443. https://doi.org/10.1080/14697017.2013.851953

Meng, X. H., Zeng, S. X., Shi, J. J., Qi, G. Y., \& Zhang, Z. B. (2014). The relationship between corporate environmental performance and environmental disclosure: An empirical study in China. Journal of Environmental Management, 145, 357-367. https://doi. org/10.1016/j.jenvman.2014.07.009

Organização das Naçôes Unidas no Brasil (ONU Brasil). (2015). Naçóes Unidas Brasil. Recuperado de http:// www.nacoesunidas.org/acao/meioambiente/. [Consulta: 15 de setembro de 2019].

Programa das Naçóes Unidas para o Desenvolvimento (PNUD). (2019). Objetivos do Desenvolvimento Sustentável. Recuperado de http://www.br.undp.org/content/brazil/pt/home/sustainable-development-goals. html. [Consulta: 20 de abril de 2019].

Rosa, F.S, Guesser, T., Hein, N., Pfitscher, E. D., \& Lunkes, R. J. (2015). Environmental impact management of
Brazilian companies: Analyzing factors that influence disclosure of waste, emissions, effluents, and other impacts. Journal of Cleaner Production, 96, 148-160. https://doi.org/10.1016/j.jclepro.2013.10.035

Rosati, F., \& Faria, L. G. (2019). Addressing the SDGs in sustainability reports: The relationship with institutional factors. Journal of Cleaner Production, 215, 13121326. https://doi.org/10.1016/j.jclepro.2018.12.107

Said, R., Omar, N., \& Abdullah, W. N. (2013). Empirical investigations on boards, business characteristics, human capital and environmental reporting. Social Responsibility Journal, 9(4), 534-553. https:/doi. org/10.1108/SRJ-02-2012-0019

Schio, N. S., Mazzioni, S., Moura, G. D., \& Dal Magro, C. B. (2019). Objetivos de Desenvolvimento Sustentável e as empresas participantes do mercado acionário brasileiro. Anais USP Internacional Conference in Accounting. São Paulo. Recuperado de https://congressousp. fipecafi.org/anais/19UspInternational/congressaoconsultar-trabalho-por-titulo.html.pdf [Consulta: 15 de outubro de 2019].

Schönherr, N., Findler, F., \& Martinuzzi, A. (2017). Exploring the interface of CSR and the sustainable development goals. Transnational Corporations, 24(3), 33-47. https://doi.org/10.18356/d3e73f33-en

Souza, M. A., Rásia, K. A., \& Jacques, F. V. (2010). Evidenciação de informações ambientais pelas empresas integrantes do índice de sustentabilidade empresarialISE. Revista de Contabilidade e Controladoria, 2(1), 51-139.

Suttipun, M., \& Stanton, P. (2012). Determinants of Environmental Disclosure in Thai Corporate Annual Reports. International Journal of Accounting and Financial Reporting, 2(1), 99-115. https://doi.org/10.5296/ ijafr.v2i1.1458

United Nations. (2018). Sustainable Development Goals. Recuperado de https://www.un.org/sustainabledevelopment [Consulta: 05 de fevereiro de 2019]. 
Vieira, K. M., Velasquez, M. D., Losekann, V. L., \& Ceretta, P. S. (2011). A influência da governança corporativa no desempenho e na estrutura de capital das empresas listadas na Bovespa. Revista Universo Contábil, 7(1), 46-67. https://doi.org/10.4270/ruc.2011104

Vilhena, F. A. C., \& Camargos, M. A. (2015). Governança corporativa, criação de valor e desempenho econômico-financeiro: evidências do mercado brasileiro com dados em painel, 2005-2011. REGE-Revista de Gestão, 22(1), 77-96. https://doi.org/10.5700/rege552

World Business Council for Sustainable Development (WBCSD). (2021). wbcsd. Recuperado de www. wbcsd.org [Consulta: 20 de fevereiro de 2019].

World Commission on Environment and Development (WCED). (1987). Our Common Future. Report of the World Commission on Environment and Development (Brundtland Report). United Nations.
Xia, B., Olanipekun, A., Chen, Q., Xie, L., \& Liu, Y. (2018). Conceptualising the state of the art of corporate social responsibility (CSR) in the construction industry and its nexus to sustainable development. Journal of Cleaner Production, 195, 340-353. https:// doi.org/10.1016/j.jclepro.2018.05.157

Fecha de recepción: 18 de octubre de 2020 Fecha de aceptación: 24 de mayo de 2021 Correspondencia: francielipacassa@unochapeco.edu.br sady@unochapeco.edu.br crisbau@unochapeco.edu.br andrecmed@unochapeco.edu.br 